Fig. 4. (A to D) Uptake of $\left[{ }^{35}\right.$ S]DMSP (top, circles) and $\left[{ }^{14} \mathrm{C}\right] \mathrm{GBT}$ (bottom, circles) by axenic cultures of $T$. pseudonana (left) and $T$. oceanica (right). Solid and open symbols correspond to dark and light incubations, respectively. Time series of isotope uptake in the presence of potential competitive inhibitors, $10 \mu \mathrm{M}$ of non-radio-labeled DMSP angles) are also shown. Error bars correspond to standard deviation from triplicate measurements. (squares), and $10 \mu \mathrm{M} \mathrm{GBT}$ (tri-

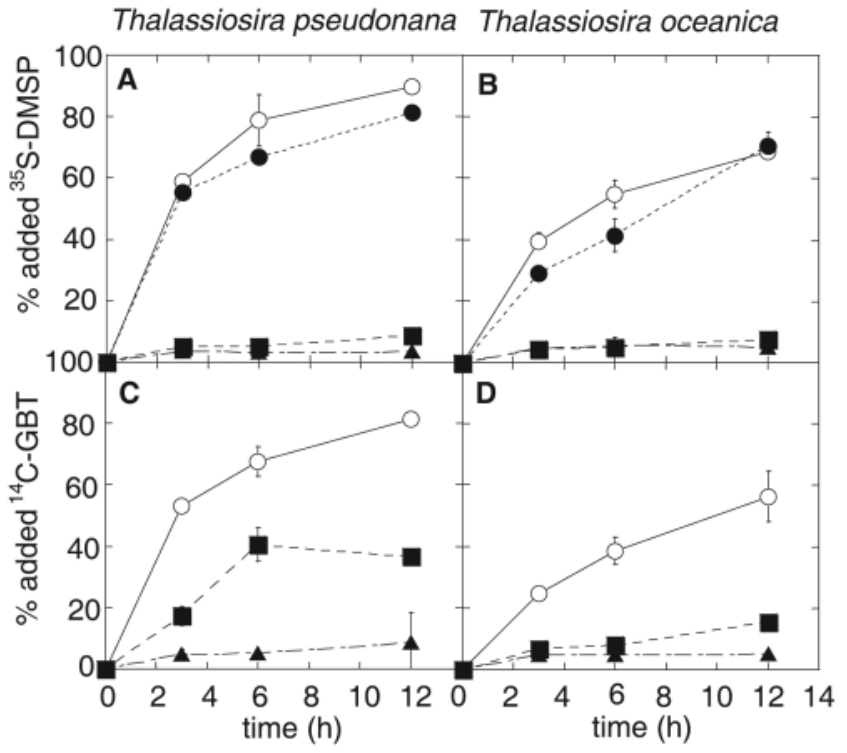

taxa $(19,30)$, further reveals how metabolically versatile phytoplankton are as a fundamental ecological player in the ocean and how challenging it becomes to implement their dynamics in oceanic biogeochemical models.

\section{References and Notes}

1. G. Malin, G. O. Kirst, J. Phycol. 33, 889 (1997).

2. J. Stefels, J. Sea Res. 43, 183 (2000).

3. D. C. Yoch, Appl. Environ. Microbiol. 68, 5804 (2002).

4. W. Sunda, D. J. Kieber, R. P. Kiene, S. Huntsman, Nature 418, 317 (2002)

5. R. J. Charlson, J. E. Lovelock, M. O. Andreae, S. G. Warren Nature 326, 655 (1987).

6. R. Simó, Trends Ecol. Evol. 16, 287 (2001).

7. M. O. Andreae, P. J. Crutzen, Science 276, 1052 (1997).

8. R. Simó, C. Pedrós-Alió, Nature 402, 396 (1999).

9. R. P. Kiene, L. J. Linn, J. A. Bruton, J. Sea Res. 43, 209 (2000).
10. R. P. Kiene, L. ]. Linn, ]. M. González, M. A. Moran, ]. A. Bruton, Appl. Environ. Microbiol. 65, 4549 (1999).

11. R. Simó, S. D. Archer, C. Pedrós-Alió, L. Gilpin, C. E. Stelfox-Widdicombe, Limnol. Oceanogr. 47, 53 (2002).

12. R. Simó, Can. J. Fish. Aquat. Sci. 5, 673 (2004).

13. R. R. Malmstrom, R. P. Kiene, D. L. Kirchman, Limnol. Oceanogr. 49, 597 (2004).

14. M. Vila et al., Appl. Environ. Microbiol. 70, 4648 (2004).

15. R. R. Malmstrom, R. P. Kiene, M. Vila, D. L. Kirchman, Limnol. Oceanogr. 50, 1924 (2005).

16. B. Kempf, E. Bremer, Arch. Microbiol. 170, 319 (1998).

17. R. P. Kiene, L. P. Hoffmann Williams, J. E. Walker, Aquat. Microb. Ecol. 15, 39 (1998).

18. G. Rocap et al., Nature 424, 1042 (2003).

19. J. A. Raven, Limnol. Oceanogr. 42, 198 (1997).

20. E. V. Armbrust et al., Science 306, 79 (2004).

21. Transporter Protein Analysis Database, www.membranetransport.org.
22. M. D. Keller, W. K. Bellows, R. R. L. Guillard, in Biogenic Sulfur in the Environment, E. Saltzman, W. ]. Cooper, Eds. (American Chemical Society, Washington, DC, 1989), pp. 167-182.

23. M. Corn et al., in Biological and Environmental Chemistry of DMSP and Related Sulfonium Compounds, R. P. Kiene, P. T. Visscher, M. D. Keller, G. O. Kirst, Eds. (Plenum, New York, 1996).

24. S. Belviso, H. Claustre, ]. C. Marty, Limnol. Oceanogr. 46, 989 (2001)

25. F. Partensky, ]. Blanchot, D. Vaulot, Bull. Inst. Oceanogr. (Monaco) 19, 457 (1999).

26. V. Smetacek, R. Scharek, E. M. Nöthig, in Antarctic Ecosystems Ecological Change and Conservation, K. R. Kerry, G. Hempel, Eds. (Springer, Berlin, 1990), pp. 103-114.

27. H. W. Paerl, Appl. Environ. Microbiol. 57, 473 (1991).

28. L. R. Moore, A. F. Post, G. Rocap, S. W. Chisholm, Limnol. Oceanogr. 47, 989 (2002).

29. M. V. Zubkov, B. M. Fuchs, G. A. Tarran, P. H. Burkill, R. Amann, Appl. Environ. Microbiol. 69, 1299 (2003).

30. R. I. Jones, Mar. Microb. Food Webs 8, 87 (1994).

31. We thank C. Pedrós-Alió for providing

microautoradiography advice and critical discussions, ]. M. González for assistance with the Prochlorococcus genome, F. Unrein and L. Arín for help with the identification of phototrophic cells under the microscope, and E. Blanch and J. Felipe for assistance with flow cytometry cell sorting. This research was supported by the European Union through project Bacterial Single-Cell Approaches to the Relationship Between Diversity and Function in the Sea (BASICS, EVK3-CT-2002-00078), by the Spanish Ministry of Education and Science through projects Relevancia de la diversidad microbiana filogenética y de estados fisiológicos para los procesos biogeoquímicos marinos (MicroDiFF, REN2001-2120/MAR to J.M.G.) and Ciclo del Azufre en el Oceano Superficial (CAOS, CTM200420022-E to R.S.) and a Ph.D. studentship to M.V.-C., and by a Catalan government grant (2005SGR00021 to R.S.).

\section{Supporting Online Material}

www.sciencemag.org/cgi/content/full/314/5799/652/DC1

Materials and Methods

Fig. S1

Tables S1 and S2

8 June 2006; accepted 19 September 2006 10.1126/science. 1131043

\title{
A Centrosome-Independent Role for $\gamma$-TuRC Proteins in the Spindle Assembly Checkpoint
}

Hannah Müller, Marie-Laure Fogeron, Verena Lehmann, Hans Lehrach, Bodo M. H. Lange*

The spindle assembly checkpoint guards the fidelity of chromosome segregation. It requires the close cooperation of cell cycle regulatory proteins and cytoskeletal elements to sense spindle integrity. The role of the centrosome, the organizing center of the microtubule cytoskeleton, in the spindle checkpoint is unclear. We found that the molecular requirements for a functional spindle checkpoint included components of the large $\gamma$-tubulin ring complex ( $\gamma$-TuRC). However, their localization at the centrosome and centrosome integrity were not essential for this function. Thus, the spindle checkpoint can be activated at the level of microtubule nucleation.

$\mathrm{T}$ he classical function of the centrosome is the organization of microtubules in higher eukaryotic cells. Its duplication and function are tightly integrated into cell cycle regulatory processes $(1,2)$. A role for the centrosome in the spindle assembly checkpoint, as an essential guardian of cell cycle progression, has been suggested but not established on the molecular level (3). $\gamma$-tubulin is a highly conserved component of the microtubule-organizing center (MTOC) in most animal cells and is involved in the initiation of microtubule nucleation $(4,5), \gamma$-tubulin is mainly found in two complexes: the large $\gamma$-tubulin ring complex
( $\gamma$-TuRC) (comprising Grip71, Grip75, Grip84, Grip91, Grip128, Grip163, and $\gamma$-tubulin in Drosophila) and its subunit, called the $\gamma$-tubulin small complex ( $\gamma$-TuSC) (comprising Grip84, Grip91, and $\gamma$-tubulin) $(6,7) \cdot \gamma$-TuRC promotes the nucleation of a microtubule filament $(4,6,8)$. In addition, $\gamma$-tubulin is thought to be required for a $\mathrm{G}_{1}$-related checkpoint pathway and spindle formation (9-12). Finally, the centrosome-associated fraction of $\gamma$-tubulin ring proteins is essential for coordinating mitotic events (13-15).

We investigated the role of core centrosomal proteins such as $\gamma$-TuRC proteins and centrosomin (cnn) (16) in spindle checkpoint activation in Drosophila cells (supporting online material text) by depleting target proteins using RNA interference (RNAi) (17) (Fig. 1). We focused on the analysis of the $\gamma$-TuSC components $\gamma$-tubulin and Grip84, the $\gamma$-TuRC component Grip71, and the

Department of Vertebrate Genomics, Max-Planck Institute for Molecular Genetics, Ihnestrasse 73, D-14195 Berlin, Germany.

*To whom correspondence should be addressed. E-mail: lange_b@molgen.mpg.de 
centrosomal core protein $\mathrm{cnn}$, which is not part of the $\gamma$-TuRC. Cells were transfected with double-stranded RNA (dsRNA) for these components and for enhanced green fluorescent protein (EGFP) as a negative control and were harvested for immunofluorescence microscopy and immunoblotting experiments. The RNAi experiments reduced the levels of target proteins (Grip71, $\gamma$-tubulin, and $\mathrm{cnn}$ ) by $>90 \%$ (Fig. 1A). The cells treated with dsRNA for Grip84, Grip71, and $\gamma$-tubulin showed a clear increase in the number of mitotic cells as opposed to the control cells (Fig. 1B). In contrast, the mitotic index of cells depleted of cnn did not significantly differ from that of the control cells (Fig. 1B).

To elucidate possible mechanisms of mitotic arrest, we defined defects in spindle morphology and microtubule organization. Normal bipolar biastral microtubule organization was observed in control cells (Fig. 1, C and D). The cells treated with dsRNA for $\gamma$-tubulin and Grip84 exhibited bipolar monastral (Fig. 1, E and F) and monopolar anastral (Fig. 1G) spindle organization with metaphase-like chromosome arrangements. Cells in the $\gamma$-TuRC knockdown experiments predominately formed spindles with one centrosome (Fig. 1, E to G). In contrast, cnn depletion led to anastral bipolar spindle arrangements, with anastral poles that were frequently barrel-shaped and unfocused (Fig. 1, H and I). This observation indicated the absence of intact mitotic centrosomes, as verified by immunofluorescence microscopy (Fig. 1, H and I). In spite of these abnormalities, anaphase progressed normally in cnn-depleted cells (Fig. 1H) without an increase of abnormal chromosome arrangement when compared to control cells. This finding is consistent with the fact that the removal of centrosomes does not prevent cells from building a functional spindle and progressing to anaphase (18-20). Thus, the depletion of $\gamma$-TuRC compo-
A Grip71-

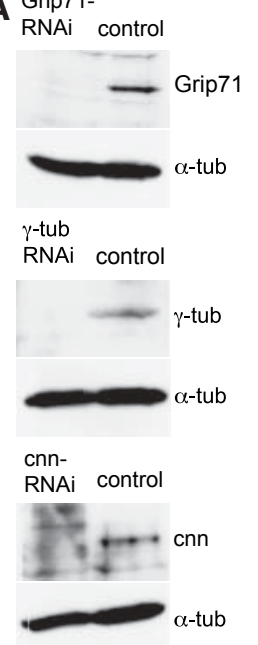

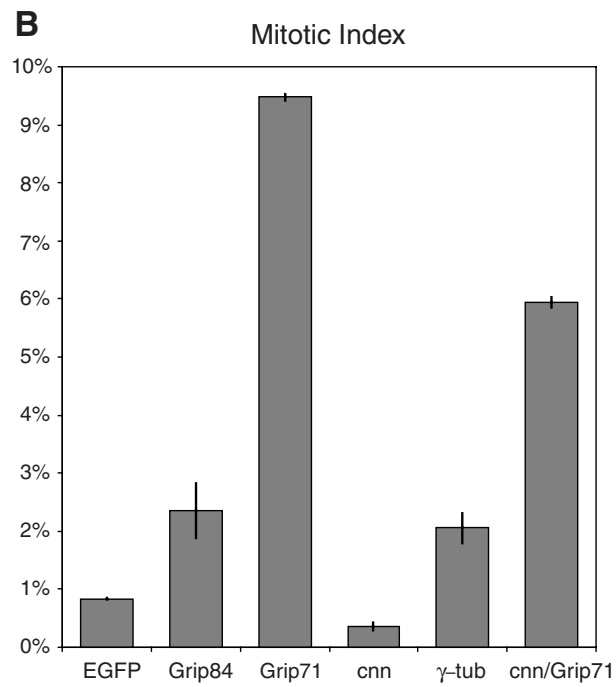

Fig. 1. Depletion of $\gamma$-TuRC proteins by means of RNAi leads to abnormal spindle organization, aberrant centrosome number, and increased mitotic index in SL2 cells. (A) Depletion of Grip71, $\gamma$-tubulin ( $\gamma$-tub), and cnn was confirmed by immunoblotting. $\alpha$-tubulin ( $\alpha$-tub) was used as a loading control. (B) The mitotic index was quantified by immunofluorescence microscopy with the use of an antibody to phosphorylated histone 3. The mitotic index was elevated after the knockdown of $\gamma$-TuRC components and the simultaneous knockdown of cnn and Grip71, when compared to EGFP negative control experiments. Depletion of $\mathrm{cnn}$ alone did not affect the mitotic index. Error bars indicate SD. (C to J) Immunofluorescence microscopy of dsRNA-transfected SL2 cells, labeled with an antibody to $\alpha$-tubulin (green), antibody to CP190 (red) $[(C),(D)$, and (J)], antibody to $\mathrm{cnn}(\mathrm{red})[(\mathrm{E})$ to $(\mathrm{G})]$, and antibody to $\gamma$-tubulin (red) $[(\mathrm{H})$ and $(\mathrm{I})]$. DNA was labeled with 4',6'-diamidino-2-phenylindole (DAPI) dihydrochloride (blue) [(C) to (J)]. Normal bipolar spindle formation and centrosome number in EGFP control cells are shown in (C) and (D). Reduction of levels of $\gamma$-TuRC proteins (Grip84 and $\gamma$-tubulin) resulted in bipolar monoastral $[(E)$ and $(F)]$ and anastral monopolar (G) spindles with one centrosome per cell and an amphitelic-like chromosome arrangement. Cells transfected with cnndsRNA [(H) and $(\mathrm{I})]$ and cnn-dsRNA in combination with Grip71-dsRNA (J) were lacking centrosomes at the spindle poles. Scale bar in (J), $5 \mu \mathrm{m}$, for (C) to (J).
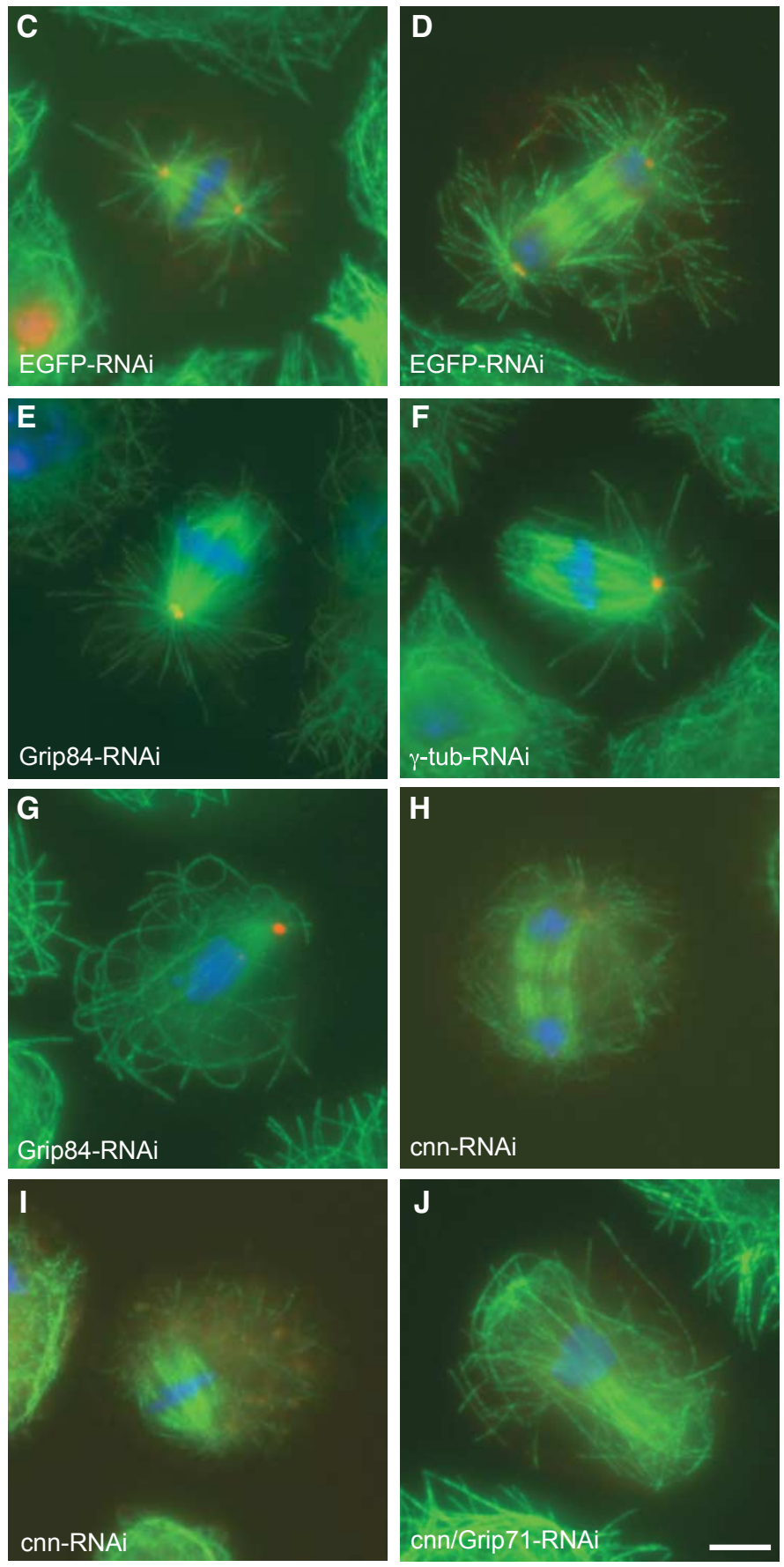
Fig. 2. (A) Coimmunoprecipitation experiments show that $\gamma$-tubulin, Cdc20, and BubR1 were in a complex. Immunoblotting analyses of immunopurified (IP) $\gamma$-tubulin from Drosophila (DM) embryonic extract (left panel) and tandem affinity purified (TAP) $\gamma$-tubulin from human (HS) cell extract (right panel) are shown. $\gamma$-tubulin was purified as detected with the antibody to $\gamma$-tubulin in both experiments. In the TAP experiments, the endogenous $\gamma$-tubulin as well as the larger $\gamma$-tubulin fusion protein with the calmodulin-binding site (cbs- $\gamma$-tub) were detected. BubR1 and Cdc20 were copurified with $\gamma$-tubulin. The mock purifications (mock) with the use of either nonimmune rabbit immunoglobins (left panel) or human immunoglobins and calmodulin beads (right panel) were negative. Drosophila embryonic or human cell extracts (Extr.) were used as positive controls for antibody labeling. (B) Depletion of $\gamma$-tubulin activates the spindle checkpoint, whereas the double knockdown confirms that $\gamma$-tubulin and Cdc20 functionally interact. The mitotic index of Drosophila SL2 cells

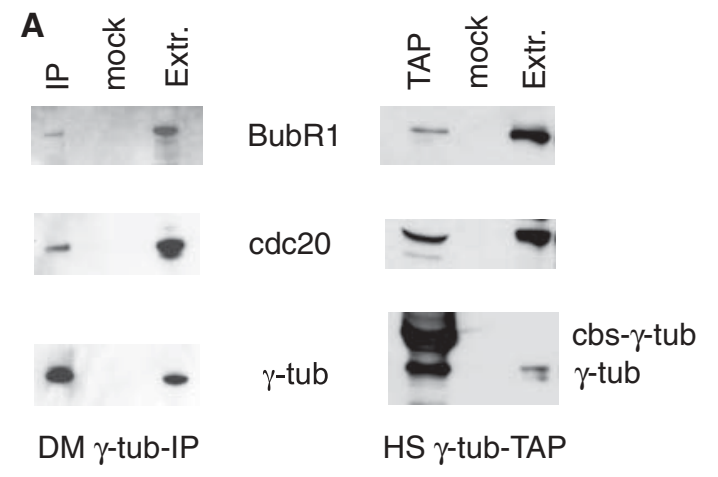

B Mitotic Index

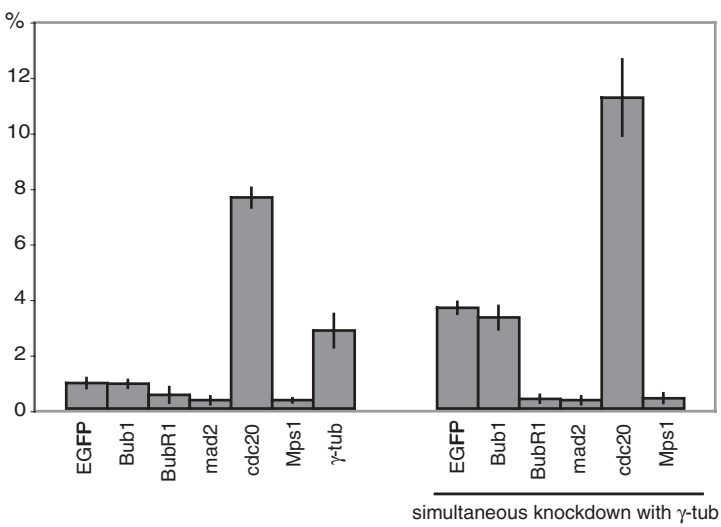

was determined by immunofluorescence microcopy after single (left) or simultaneous (right) depletion experiments. Depletion of $\gamma$-tubulin and Cdc20 led to an increased mitotic index when compared to negative control cells. The simultaneous knockdown of $\mathrm{Cdc} 20$ with $\gamma$-tubulin resulted in an accumulative increase of mitotic cells. In contrast, the simultaneous depletion of checkpoint kinases (except Bub1) with $\gamma$-tubulin resulted in a reduced mitotic index in comparison to the EGFP/ $\gamma$-tubulin control. Error bars indicate SD.
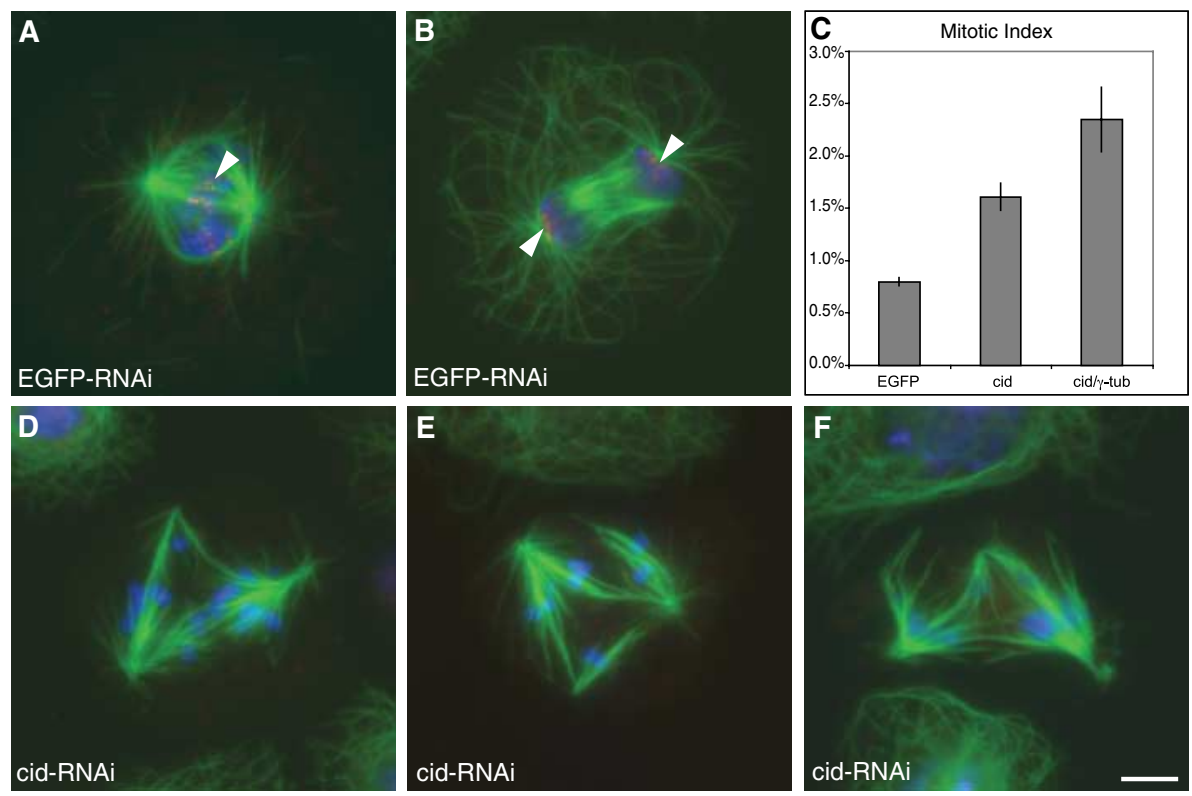

Fig. 3. RNAi of CID, an inner kinetochore protein, causes mitotic arrest and aberrant spindle formation and chromosome arrangements in Drosophila SL2 cells. (A and B) Labeling of control cells with antibodies to CID and $\alpha$-tubulin detected a distinct kinetochore structure and normal microtubule organization during different mitotic stages. Arrowheads indicate CID labeling (red/yellow). DNA was labeled with DAPI (blue) (C) In comparison to control cells, depletion of CID by means of RNAi triggered a mitotic arrest that was increased further through double knockdown with $\gamma$-tubulin. Error bars indicate SD. (D to F) In immunofluorescence microscopy, CID was not detected after RNAi, demonstrating its efficient depletion from the kinetochore. The frequent metaphase arrest and displaced chromosomes indicated the microtubule-kinetochore attachment defect. Scale bar in (F), $5 \mu \mathrm{m}$, for (A), (B), and (D) to (F).

nents triggers mitotic arrest and impedes centrosome separation or duplication, whereas the removal of the centrosomal core protein cnn does not.

This discovery raised the questions of (i) how the depletion of $\gamma$-tubulin could activate arrest and whether this specific trigger (ii) requires an intact centrosome or (iii) is independent of centrosome localization. To address these questions, we depleted $\gamma$-TuRC proteins in the absence of an intact MTOC structure.

The simultaneous knockdown of cnn and Grip71 triggered mitotic arrest in the absence of a detectable centrosome (Fig. 1J). Thus, an intact centrosome is not a prerequisite for the $\gamma$-TuRCmediated mitotic arrest. This result suggested at least two explanations: The depletion of microtubule nucleation factors will probably lead to a reduced number of microtubules. As a consequence, microtubule-kinetochore attachment is more likely to be absent and thus trigger the spindle checkpoint (21). Furthermore, $\gamma$-tubulin might be part of a signaling complex that actually activates the checkpoint when depleted.

Our hypothesis is that the reduction of microtubule density is not the sole trigger for mitotic arrest, because overall microtubule density was reduced in only a fraction of the arrested cells (Fig. 1G); most mitotically arrested cells in the $\gamma$-TuRC RNAi experiment showed abundant microtubule arrays with amphitelic-like chromosome microtubule attachment (Fig. 1, E and F). Thus, we tested whether the $\gamma$-TuRC components might be part of a signaling complex, triggering the spindle checkpoint when $\gamma$-TuRC proteins were abrogated. Checkpoint components are $\mathrm{Cdc} 20$, as part of the anaphase-promoting complex, regulating sister chromatid separation; and the kinases BubR1, Bub1, Mad2, and Mps1, which have been proposed to sense the occupancy or lack of tension at the microtubule plus ends (22).

We investigated the possible interaction of spindle assembly checkpoint components with $\gamma$-TuRC both on a functional and on a biochemical level, using RNAi, immunoprecipitation (IP), and tandem affinity purification (TAP) approaches (17). We purified $\gamma$-tubulin complexes by IP from Drosophila preblastoderm extracts and by TAP from mitotically synchronized human cells (fig. S1). Biochemical analysis of the complexes revealed that $\gamma$-tubulin was in a complex with BubR1 and Cdc20 in Drosophila and human cells (Fig. 2A), demonstrating an evo- 
lutionarily conserved association of $\mathrm{Cdc} 20$ and BubR1 with $\gamma$-TuRC components. The molecular interaction data, from both Drosophila and human cells, suggested a mechanism coupling the spindle assembly checkpoint to $\gamma$-TuRC.

Because BubR1 and Cdc20 were in a complex with $\gamma$-tubulin, we investigated the functional importance of this interaction for the activation of the spindle checkpoint. A true spindle checkpoint protein is described as a component required for the activation of the checkpoint (22). In its absence, cells do not arrest in metaphase but separate sister chromatids and then exit mitosis $(23,24)$. We used this property of the spindle checkpoint kinases to test whether depletion of $\gamma$-tubulin actually triggers a true spindle checkpoint or leads to an increase of the mitotic index through another pathway. The simultaneous knockdown of $\gamma$-tubulin and either of the checkpoint kinases described above had (apart from Bub1) a much-reduced mitotic index when compared to the EGFP/ $\gamma$-tubulin control knockdown (Fig. 2B). This means that these checkpoint proteins were necessary for the $\gamma$-tubulin depletion-mediated mitotic arrest, confirming that $\gamma$-tubulin triggered a proper spindle assembly checkpoint response. As expected, Bub1 did not show any significant difference in the mitotic rate when compared to the negative control $\mathrm{EGFP} / \gamma$-tubulin knockdown (Fig. 2B) (25). In addition, the simultaneous knockdown between Cdc20 and $\gamma$-tubulin increased the percentage of mitotic cells when compared to the $\mathrm{Cdc} 20$ single knockdown (Fig. 2B). Thus, the effects of $\gamma$-tubulin and Cdc20 depletion are cumulative, and $\gamma$-tubulin and Cdc20 interact functionally, which agrees with our biochemical data that $\gamma$-tubulin and $\mathrm{Cdc} 20$ are in a complex.

The kinetochore is composed of transiently associated proteins such as the checkpoint proteins, as well as more structural proteins [for example, CENP-B, CENP-C, and the histone H3-related protein CENP-A (CID in Drosophila)] (26). To test the respective contribution of signaling to the checkpoint from $\gamma$-TuRC and from the kinetochore, we removed a structural component of the kinetochore in SL2 cells. We achieved this step by using RNAi of the Drosophila CENP-A homolog CID (Fig. 3), a protein that is localized in or close to the inner plate of the Drosophila kinetochore and is required for kinetochore assembly (27). The efficient depletion of CID (Fig. 3, D to F) as compared to control cells (Fig. 3, A and B) caused mitotic arrest (Fig. 3C) and displaced the chromosomes from the metaphase plate (Fig. 3, $\mathrm{D}$ to F), suggesting microtubule-kinetochore attachment defects. Knockdown of $\gamma$-tubulin together with CID caused a further increase in the mitotic index when compared to single CID knockdown (Fig. 3C). This supports our hypothesis that $\gamma$-TuRC depletion is likely to activate an additional spindle checkpoint signal.

It has been suggested that the centrosome serves as a complex platform for multiple cellular signaling pathways $(2,3,28)$. For example, cyclin B degradation, catalyzed through $\mathrm{Cdc} 20$, starts on the centrosome, thus functioning as a molecular hub integrating the interaction of proteins that regulate mitotic progression $(28-30)$. Here we provide evidence that $\gamma$-TuRC proteins, rather than the centrosome per se, play a molecular role in the activation of the spindle checkpoint. We propose that $\gamma$-TuRC proteins are integrated in a signaling mechanism at the microtubule minus ends, and they are interacting with spindle checkpoint components independently of centrosome integrity.

\section{References and Notes}

1. M. F. Tsou, T. Stearns, Curr. Opin. Cell Biol. 18, 74 (2006).

2. G. Sluder, Nat. Rev. Mol. Cell Biol. 6, 743 (2005).

3. S. Doxsey, W. Zimmerman, K. Mikule, Trends Cell Biol. 15, 303 (2005).

4. D. Job, O. Valiron, B. Oakley, Curr. Opin. Cell Biol. 15, 111 (2003).

5. M. Moritz, D. A. Agard, Curr. Opin. Struct. Biol. 11, 174 (2001).

6. Y. Zheng, M. L. Wong, B. Alberts, T. Mitchison, Nature 378, 578 (1995).

7. K. Oegema et al., J. Cell Biol. 144, 721 (1999).

8. M. Moritz, M. B. Braunfeld, V. Guenebaut, ]. Heuser, D. A. Agard, Nat. Cell Biol. 2, 365 (2000).

9. B. R. Oakley, Curr. Top. Dev. Biol. 49, 27 (2000).

10. S. Doxsey, D. McCollum, W. Theurkauf, Annu. Rev. Cell Dev. Biol. 21, 411 (2005).

11. L. Vardy, T. Toda, EMBO J. 19, 6098 (2000).

12. C. Verollet et al., J. Cell Biol. 172, 517 (2006).

13. N. L. Prigozhina et al., Mol. Biol. Cell 15, 1374 (2004).

14. V. Barbosa, M. Gatt, E. Rebollo, C. Gonzalez, D. M. Glover, J. Cell Sci. 116, 929 (2003).

15. N. Colombie et al., Mol. Biol. Cell 17, 272 (2006).

16. K. Li, T. C. Kaufman, Cell 85, 585 (1996).

17. Materials and methods are available as supporting material on Science Online.

18. A. Khodjakov, C. L. Rieder, J. Cell Biol. 153, 237 (2001).
19. E. H. Hinchcliffe, F. J. Miller, M. Cham, A. Khodjakov, G. Sluder, Science 291, 1547 (2001).

20. T. L. Megraw, L. R. Kao, T. C. Kaufman, Curr. Biol. 11, 116 (2001).

21. C. L. Rieder, A. Schultz, R. Cole, G. Sluder, J. Cell Biol. 127, 1301 (1994).

22. A. Musacchio, K. G. Hardwick, Nat. Rev. Mol. Cell Biol. 3, 731 (2002).

23. G. J. Kops, D. R. Foltz, D. W. Cleveland, Proc. Natl. Acad. Sci. U.S.A. 101, 8699 (2004)

24. G. K. Chan, S. A. Jablonski, V. Sudakin, J. C. Hittle, T. J. Yen, J. Cell Biol. 146, 941 (1999).

25. M. Bettencourt-Dias et al., Nature 432, 980 (2004).

26. G. K. Chan, S. T. Liu, T. J. Yen, Trends Cell Biol. 15, 589 (2005).

27. M. D. Blower, G. H. Karpen, Nat. Cell Biol. 3, 730 (2001).

28. J. W. Raff, K. Jeffers, ]. Y. Huang, J. Cell Biol. 157, 1139 (2002).

29. C. L. Rieder, S. Faruki, A. Khodjakov, Trends Cell Biol. 11, 413 (2001).

30. B. M. H. Lange, Curr. Opin. Cell Biol. 14, 35 (2002).

31. We acknowledge R. Aebersold, I. A. Dawson, G. Goshima, A. C. Gringas, G. Karpen, T. Kaufman, C. Sunkel, R. Vale, $W$. Whitfield, and $Y$. Zheng for the gift of antibodies, cells, and DNA constructs; the help of $S$. Haesler and D. Weichart with parts of the fluorescence-activated cell sorting and IP experiments; and our colleagues and A. Ploubidou for their comments on the manuscript. The work in the laboratory of B.M.H.L. is funded through the Berliner Senat für Kultur, Wissenschaft und Forschung, Project Ultrastructural Network, European Funds for Regional Development; by the Bundesministerium fur Bildung und Forschung, German Network for Genome Research 2, Systematic-Methodological Platform-Protein; by the European Union; and by the Max-Planck Society and the Thyssen Foundation.

\section{Supporting Online Material}

www.sciencemag.org/cgi/content/ful//314/5799/654/DC1 Materials and Methods

SOM Text

Figs. S1 to S3

References

21 July 2006; accepted 18 September 2006

$10.1126 /$ science 1132834

\title{
Odorant Receptor-Derived cAMP Signals Direct Axonal Targeting
}

\author{
Takeshi Imai, ${ }^{1,2}$ Misao Suzuki, ${ }^{3}$ Hitoshi Sakano ${ }^{1,2 *}$
}

In mammals, odorant receptors (ORs) direct the axons of olfactory sensory neurons (OSNs) toward targets in the olfactory bulb. We show that cyclic adenosine monophosphate (cAMP) signals that regulate the expression of axon guidance molecules are essential for the OR-instructed axonal projection. Genetic manipulations of ORs, stimulatory G protein, CAMP-dependent protein kinase, and CAMP response element-binding protein shifted the axonal projection sites along the anteriorposterior axis in the olfactory bulb. Thus, it is the OR-derived cAMP signals, rather than direct action of OR molecules, that determine the target destinations of OSNs.

$\mathrm{E}$ ach olfactory sensory neuron (OSN) in the mouse expresses only one functional odorant receptor (OR) gene out of $\sim 1000$ members (1-3). Axons from OSNs expressing a given OR converge onto a specific site, the glomerulus, in the olfactory bulb (4-6). It has been proposed that OR molecules at axon termini may directly recognize guidance cues on the olfactory bulb and mediate homophilic interactions of like axons $(6-10)$. OR molecules are heterotrimeric guanine nucleotide-binding protein (G protein)-coupled receptors (GPCRs) that transduce the odorant-binding signals by activating the olfactory-specific $G$ protein $\left(G_{o l f}\right)$ expressed in mature OSNs. The activation of $\mathrm{G}_{\mathrm{olf}}$ stimulates adenylyl cyclase type III, generating cAMP, which opens cyclic nucleotidegated $(\mathrm{CNG})$ channels. Mice deficient for $\mathrm{G}_{\text {olf }}$ and CNGA2 are anosmic but form a normal glomerular map (11-13), which suggests that a 